\title{
Identification of Contact Formations: Resolving Ambiguous Force Torque Information
}

\author{
Katharina Hertkorn, Maximo A. Roa, Carsten Preusche, Christoph Borst, Gerd Hirzinger
}

\begin{abstract}
This paper presents the identification of contact formations using force torque information. As force torque measurements do not map uniquely to their corresponding contact formations, three steps are performed: Initially, the wrench space for each contact formation is computed automatically. Then, a contact formation graph is augmented with a similarity index that reflects the similarity of contact formations with respect to their spanned wrench spaces. A particle filter is used to represent the likeliness of a contact formation given a force torque measurement. Finally, this probability distribution is resolved taking the similarity index, the transitions of the contact formation graph and the history of identified contact formations into account. This allows the recognition of the order of demonstrated contact formations by a measured set of forces and torques. The approach is verified by experiments.
\end{abstract}

\section{INTRODUCTION}

In industrial assembly, an automated system that generates the robotic assembly strategy directly from the computerbased constructed object is desirable. Many assembly planning systems have shown the possibility to plan a strategy offline, e.g. based on user input or geometric information [1], [2]. Additionally, a fast and intuitive programming of the robotic assembly process is crucial as lot sizes decrease.

Xiao and Ji [3] and Thomas et al. [4] plan an assembly task using the so-called contact formation graph. The graph can be automatically generated based on geometric information, e.g. the CAD data of the involved objects, and the goal configuration of the objects. This undirected graph contains all the possible contact formations between two objects, but it does not include information about useful or unwanted contact formations, i.e. the way to achieve the goal contact formation through the graph is unknown. We propose to extract this information from human demonstration. The human is able to carry out an assembly task in a robust and fast way, especially when uncertainties are taken into account, and demonstration is an intuitive way for the human to program the robot. To analyze the human demonstration and to get the path the human takes through the contact formation graph, one needs to identify the shown contact formations.

Meeussen et al. [5] and Gadeyne et al. [6] identified contact formations with a particle filter using analytically derived wrench and twist spaces for each contact formation. Based on force, torque and velocity measurements, they estimated

The authors are with the Institute of Robotics and Mechatronics, DLR (German Aerospace Center), D-82234 Wessling, Germany.

\{firstname. lastname\}edlr.de the pose of the assembled object and the environment to derive the current contact formation in an online process.

The recognition of contact formations using only force torque signals was done by Skubic and Volz [7] with fuzzy logic and neural networks, which was generalized by Everett et al. [8]. Identifiers for the contact formations are trained with measured signals, and they are used to identify the contact formations without a geometric model of the object. Abegg et al. [9] used force torque signals to estimate contact formations as well, and verified the states with visual information. Mosemann et al. [10] (based on [11]) described and identified assembly process states using polyhedral convex cones. The ambiguity of force torque signals is avoided with restricting assumptions on the mapping between force torque signals and the contact formations. Suarez et al. [12] analyzed the contact force domains for the 2D case, including uncertainty in the computation of the domains. These domains are used as a preknowledge to resolve a certain contact formation with heuristics during execution [13]. McCarragher and Asada [14] recognized the transitions between different contact states using dynamic template matching. They model the planar assembly process dynamically and extract force profiles for each possible transition that can occur in the process, and then match those to the real time force torque signal.

The known approaches to identify assembly paths by demonstration require external sensors, e.g. cameras, calibrated poses of the robot and the object in the world [5], or a high amount of preknowledge about the assembly process [14]. The presented new approach solves this problem by using only measured force torque information at the robot tool and known CAD data of the objects. The offline estimation of demonstrated contact formations is based on a contact formation graph [4] that includes the possible poses of the assembled object for each contact formation; such graph can be obtained automatically. During the demonstration, only forces and torques are measured at the tool, which guarantees an intuitive and effortless setup for the human demonstration of the assembly task.

To resolve the ambiguous force torque information, three steps are performed: First, the wrench spaces for all contact formations in the graph are computed automatically. With a penetration based algorithm [15], the normal directions and the forces and torques for the contacts are calculated and a resulting wrench space is derived. Then, the graph is augmented with an index that reflects the similarity of the contact formations with respect to their spanned wrench spaces. As a second step, the measured forces and torques 


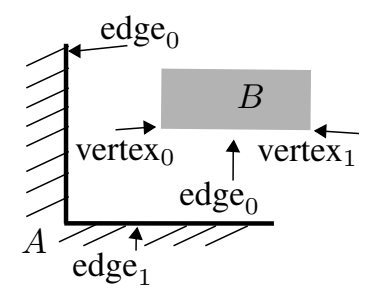

(a) Notation of the topological elements of objects

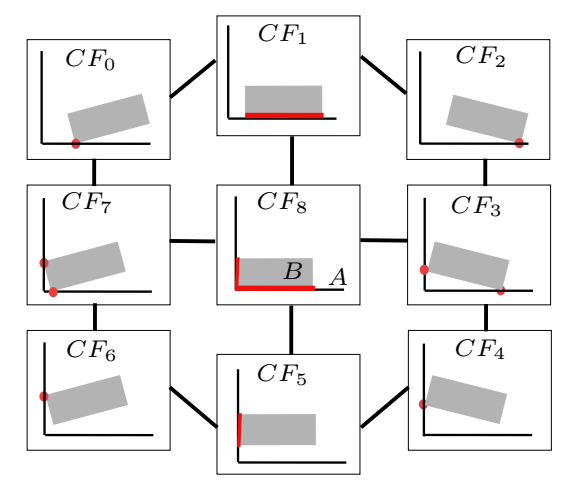

(b) Contact formation graph

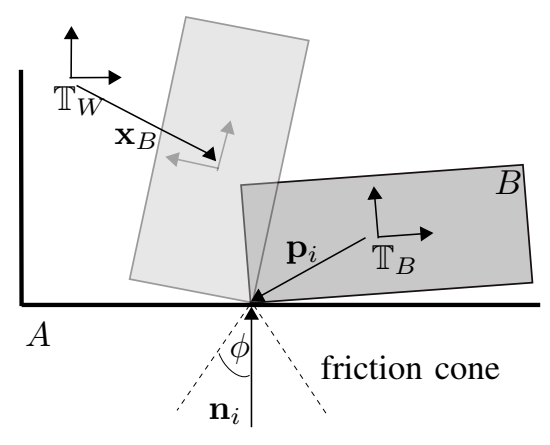

(c) Different poses of the object in the contact state $C F_{0}$ considering the same contact point.

Fig. 1. Example of a 2d-task: put a rectangle in a corner

during demonstration are mapped to their correspondent contact formation. The wrench spaces derived in step one have been previously used to identify contact formations [10]. We make the identification process more robust by considering uncertainty using a particle filter, as proposed in [5]. In contrast to [5], the wrench spaces are computed automatically and the pose of the involved objects is neither estimated nor tracked with an external camera system, as only forces and torques are measured. Of course, one force torque vector can be assigned to several contact formations due to factors such as friction. This ambiguity is reflected by the particle filter that assigns a likeliness to a certain contact formation. Finally, the third step derives the order of the shown contact formations using the similarity index to minimize the number of transitions and maximize the sum of probabilites in the graph of contact sequences.

We present the approach as follows: Section II introduces contact formation graphs. The computation of the wrench spaces for each contact in the graph is presented in Section III. A similarity index is presented in Section IV, followed by the description of a particle filter to identify the corresponding contact formation in Section V. The experimental setup and the results are described in Section VI. The paper is summarized and conclusions are drawn in Section VII.

\section{CONTACT Formation GRAPHS}

A contact formation graph is an undirected graph containing all the contact formations that can occur between two objects $A$ and $B$ [4]. We assume that object $A$ is static and object $B$ is moved, see Figure 1(a). The concepts proposed in this paper follow the definition of principal contacts between two objects proposed in [11]. A principal contact is defined as a single contact between a pair of topological elements vertex, edge or face, i.e. vertex/face, edgelface, and facelface contacts. A contact formation $C F$ is then a set of principal contacts. If a contact formation contains only one principal contact, it is called a basic contact formation.

There are $n_{C F}$ contact formations that might happen between two objects, and they are all represented by the nodes of the contact formation graph $\mathcal{C F}:=$ $\left\{C F_{0}, \ldots, C F_{n_{C F}-1}\right\}$. The edges are possible transitions
$\mathcal{T}$ between neighbor contact formations [3]: Two contact formations $C F_{i}$ and $C F_{j}$ are neighbors if either $C F_{i} \subset C F_{j}$ or $C F_{j} \subset C F_{i}$. The "no contact" state is not included in the graph. Nevertheless, it implicitly represents the state with contact forces or torques smaller than a predefined value $\epsilon$. This state is connected to all nodes in the contact formation graph.

Figure 1(b) shows a contact formation graph for a 2Dtask: put a rectangular object in a corner. There, $n_{C F}=9$ contact formations can occur with only four of them leading to the goal contact formation $C F_{8}$. The contact points in each contact formation are emphasized with red circles / lines, e.g. contact formation $C F_{0}$ represents a basic contact formation vertex $_{0} / e d g e_{1}$, and $C F_{3}$ contains two principal contacts, namely vertex $x_{0} /$ edge $_{0}$ and vertex $1 /$ edge $e_{1}$. The notation is given in Figure 1(a); in a contact formation, the topological element of the moved object is always written first.

As previously stated, the contact formation graph is generated automatically based only on geometric information [3]. For our computation, we consider that one contact state $C F_{j}$ is properly described by $n_{\mathrm{x}}$ poses of the object $B$ (object $A$ is considered static):

$$
C F_{j}=\bigcup_{k} \mathbf{x}_{B, k}, \text { with } \begin{gathered}
k \in\left[0, \ldots, n_{\mathbf{x}}-1\right] \\
j \in\left[0, \ldots, n_{C F}-1\right]
\end{gathered}
$$

where $\mathbf{x}_{B}$ is the pose of the object $B$ in the world coordinate system $\mathbb{T}_{W}$, see Figure $1(\mathrm{c})$. $\mathbb{T}_{B}$ is the coordinate system of object $B$, located in the object's center of mass.

We assume that the given poses $\mathbf{x}_{B, k}$ represent the degrees of freedom of the object during contact formation $C F_{j}$, as needed for the representation of the contact forces and torques.

\section{CONTACT Forces AND TORQUES}

This section describes a compact representation of the forces and torques that can occur in contact states between two rigid bodies $A$ and $B$. Usually, contact forces and torques are described in a world coordinate frame, but often the transformation between world and object coordinate system during measurement or robotic execution is not exactly 
known. Therefore, the representation uses the object coordinate system, thus eliminating one source of uncertainty. First, a short introduction into modeling force torque domains including fricion is given, and then a compact representation of this domain for each contact formation is proposed. The compact representation is used to compute the similarity index in Section IV based on the sampled poses for each contact formation.

Modeling the contact between different objects requires a way to include the friction between them. The main models considered in the literature are the frictionless and frictional contact, or a soft contact [16]. As friction and sensor noise occur in the real world, a frictional contact model is considered in this work.

Coulomb's friction model is used to model the contact force at the $i$-th contact point $\mathbf{p}_{i}=(x, y, z) \in \mathbb{R}^{3}$, see Figure 1(c). The corresponding normal is denoted as $\mathbf{n}_{i} \in \mathbb{R}^{3}, i \in\left[0, \ldots, n_{c}-1\right]$, with $n_{c}$ the total number of contact points between two objects. The angle $\phi$ of the corresponding friction cone is $\phi=\arctan \mu$, with $\mu$ being the friction coefficient. The friction cone is approximated by a convex cone with $n_{f c}$ sides. A force $\mathbf{f}_{i}$ lying inside the friction cone is described with

$$
\mathbf{f}_{i}=\sum_{j=0}^{n_{f c}-1} a_{i j} \hat{\mathbf{n}}_{i j}, \text { with } a_{i j}>0,
$$

where $\hat{\mathbf{n}}_{i j}$ is the unitary vector along the $j$-th edge of the convex cone at the $i$-th contact. The force $\mathbf{f}_{i}$ generates a torque $\tau_{i}$ with respect to the center of mass of the object. Both $\mathbf{f}_{i}$ and $\boldsymbol{\tau}_{i}$ are grouped in a wrench vector

$$
\boldsymbol{\omega}_{i}=\left(\begin{array}{c}
\mathbf{f}_{i} \\
\boldsymbol{\tau}_{i}
\end{array}\right)=\left(\begin{array}{c}
\mathbf{f}_{i} \\
\mathbf{p}_{i} \times \mathbf{f}_{i}
\end{array}\right)=\left[\begin{array}{c}
f_{i x} \\
f_{i y} \\
f_{i z} \\
y f_{i z}-z f_{i y} \\
z f_{i x}-x f_{i z} \\
x f_{i y}-y f_{i x}
\end{array}\right]
$$

If multiple contact points occur, the spanned wrench space WS is the convex hull $C H$ of all wrenches $\boldsymbol{\omega}_{i}$, with $n_{c}$ being the number of points in contact:

$$
\mathbf{W S}=C H\left(\boldsymbol{\omega}_{i}\right) \text {, with } i \in\left[0, \ldots, n_{c}-1\right] .
$$

Equation (3) implies that the linearized friction cone describing the possible forces applied at a frictional point $\mathbf{p}_{i}$ is mapped as a 3-dimensional subspace in the 6-dimensional wrench space:

$$
\boldsymbol{\omega}_{i}=f_{i x}\left[\begin{array}{c}
1 \\
0 \\
0 \\
0 \\
z \\
-y
\end{array}\right]+f_{i y}\left[\begin{array}{c}
0 \\
1 \\
0 \\
-z \\
0 \\
x
\end{array}\right]+f_{i z}\left[\begin{array}{c}
0 \\
0 \\
1 \\
y \\
-x \\
0
\end{array}\right]
$$

Note that for a constant contact point the three base vectors are constant and linearly independent.

Accordingly, the possible wrenches applied at the contact point can be described in two ways: Using the world coordinate frame $\mathbb{T}_{W}$ implies that the contact forces ${ }^{W} \mathbf{f}_{i}$ are constant. However, the torques ${ }^{W} \boldsymbol{\tau}_{i}$ change depending on the particular orientation of the object, as the position of the center of mass is not constant in world coordinates. This leads to a wrench ${ }^{W} \boldsymbol{\omega}_{i}$ that depends on the number of sampled poses $n_{\mathbf{x}, j}$ in a contact formation to accurately represent the resulting torques. Furthermore, the quality of the identification degrades with increasing uncertainty in the world coordinate system.

On the contrary, the description of the wrench ${ }^{B} \boldsymbol{\omega}_{i}$ in the object coordinate system leads to a constant position of the contact point relative to the center of mass, and the wrench space can be described according to (5). Additionally, measurements of the contact forces and torques are also in object coordinates, see Section VI. This allows a direct comparison of measured and computed contact forces avoiding the transformation to the (unknown) world coordinate system.

\section{The Similarity IndeX}

This section describes first a procedure to obtain a compact representation of the resulting wrenches for $2 \mathrm{D}$ and 3D contact formations. Second, the augmentation of the contact formation graph with the similarity index and then its application to the identification of contact formations is specified. The similarity index reflects the overlap between the wrenches of different contact formations. To clarify the procedure, a similarity index $\gamma_{j, i}^{f}$ considering only the friction cones is introduced. Then, the computation of a similarity index that uses force and torque information is presented.

Given the sampled poses of object $B$ for each contact formation in the graph, the contact points and frictionless contact normals need to be found. They are computed using the voxmap-pointshell algorithm [15], which calculates forces and torques based on the penetration of points in voxels. The moved object $B$ is represented by a pointshell and the static object $A$ by a voxelmap. The resulting friction cone and the corresponding wrenches are calculated using the procedure described below.

\section{A. Similarity Index Considering Only Forces}

In 2-dimensional space, objects can have one rotational degree of freedom while keeping a certain basic contact formation. With a fixed angle between the objects, e.g. $C F_{1}$, the friction cone is constant in world and object coordinate frames, and (5) can be directly applied:

$$
\boldsymbol{\omega}_{i}=f_{i x}\left[\begin{array}{c}
1 \\
0 \\
-y
\end{array}\right]+f_{i y}\left[\begin{array}{l}
0 \\
1 \\
x
\end{array}\right] \text {. }
$$

With a rotational degree of freedom, e.g $C F_{0}$ or $C F_{2}$, the possible directions of the friction cone in the object coordinate system change between two extrema that can be obtained from the sampled poses in the contact formation. Figure 2(a) depicts the two extrema for contact formation $C F_{0}$. All the possible forces applied at the contact point can 


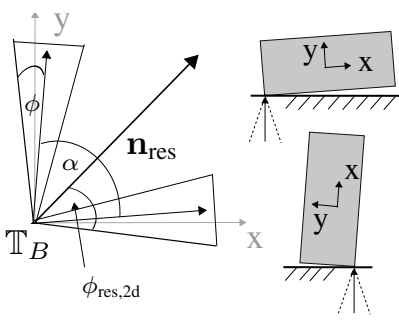

(a) Friction cones in $2 \mathrm{D}$

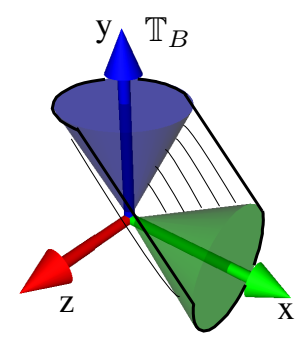

(b) Friction cones in 3D
Fig. 2. Friction cones for two DoF in 2 and 3-dimensional space

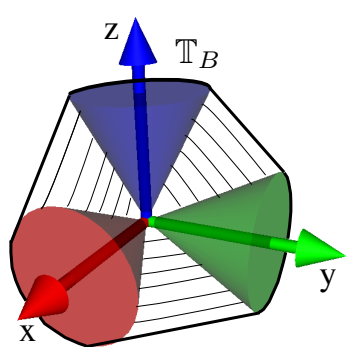

(a) Friction cones

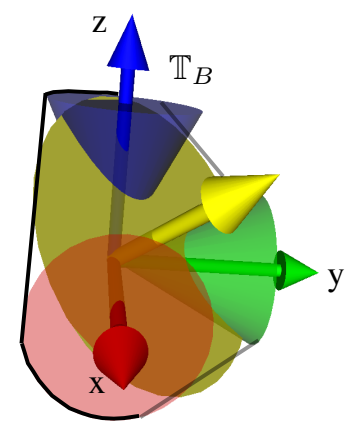

(b) Resulting friction cone
Fig. 3. Friction cones or a contact formation with three DoF in 3dimensional space

be described with one resulting friction cone. Its angle $\phi_{\text {res }, 2 \mathrm{~d}}$ is the sum of the angle $\phi$ generated by the friction coefficient and the angle $\alpha$ between the normals:

$$
\phi_{\text {res }, 2 \mathrm{~d}}=\phi+\frac{\alpha}{2} \text {. }
$$

With the resulting friction cone, all the generated wrenches at the contact point can be described using (6).

In $3 \mathrm{D}$ two interacting objects can have from zero to three rotational degrees of freedom (DoF) without changing the contact formation. Analog to the 2D case, the friction cone in object coordinates can be constant (direct application of (5)), between two or three extrema. Figure 2(b) shows an example of an $e d g e_{1} /$ face $_{1}$ contact where the friction cone is between two extrema. All possible forces that can occur for this contact formation are within the union of the two friction cones, adumbrated with the shaded area. This region can be approximated by a cone with an ellipsoid ground area. Its semi-major axis $a$ is the friction coefficient $a=\mu$ and the semi-minor axis $b$ is $b=\cos \left(\arctan (\mu)+\frac{\alpha}{2}\right)$.

Porting the exemplary contact formation $C F_{0}$ given in Figure $1(\mathrm{c})$, to the three dimensional space, it is a vertex $1 /$ face $_{1}$ contact. The friction cone is between three extrema as shown in Figure 3(a). This domain can be approximated by a resulting friction cone, see Figure 3(b). The resulting friction cone is the cone with the largest angle which is completely inscribed in the convex combination of the inital friction cones.

The approximations in 3D are evaluated using solid angles [17]. A solid angle describes the area of the spherical surface cut out by a pencil of rays starting from the same

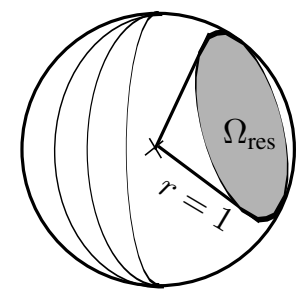

(a) Solid angle of a friction cone on a unit sphere

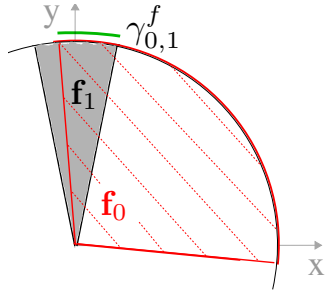

(b) Similarity index $\gamma_{0,1}^{f}$
Fig. 4. Solid angle and similarity index

point. Hence, the solid angle $\Omega_{\text {res }}$ of a convex cone equals the area of the spherical cap of a unit sphere that the cone encloses (depicted in Figure 4(a)):

$$
\Omega_{\mathrm{res}}=2 \cdot \pi \cdot\left(1-\cos \left(\phi_{\mathrm{res}, 3 \mathrm{~d}}\right)\right) .
$$

The solid angle $\Omega$ described by the original set of forces is computed using the convex hull of the set. The solid angle is then the sum of all spherical triangles on the unit sphere that the convex hull of the force set encloses. Equally, the solid angle of the resulting cone with an ellipsoidal surface area is computed. For the given example in Figure 3(b) $\Omega_{\mathrm{res}} / \Omega=0.8$ holds and for the example depicted in Figure 2(b) the ratio is 0.82 . Hence, the approximation is good enough to calculate the similarity index described in Section IV.

The similiarity index $\gamma_{j, i}$ of a contact formation $C F_{j}$ reflects the amount of overlap between its wrenches and the wrenches of its neighbor $C F_{i}$ in the graph. The previously needed projection of the friction cones on a unit sphere is now used to calculate the similarity index $\gamma_{j, i}^{f}$ : This index is the length of the intersecting arcs, depicted in Figure 4(b), for the 2-dimensional case. It is normalized with the length of the union of the two arcs. In 3-dimensional space the intersection area of two spherical caps is calculated [17] and normalized as well.

\section{B. Similarity Index Considering Forces and Torques}

As the possible wrenches generated in a contact formation describe planes or volumes in $2 \mathrm{D}$, resp. planes, volumes and hypervolumes in $3 \mathrm{D}$, the intersection between them can be a point up to a hypervolume. These intersections are not directly comparable. Therefore, an uncertainty $\boldsymbol{\eta}=$ $\left(\eta_{x}, \eta_{y}, \eta_{z}\right)^{T}$ of the location of the contact point is taken into account: $\overline{\mathbf{p}}_{i}=\mathbf{p}_{i} \pm \boldsymbol{\eta}$. The 3-dimensional subspace of (5) is then expanded to a hypervolume $\overline{\boldsymbol{\omega}}_{i}$ described by $\overline{\boldsymbol{\omega}}_{i}=C H\left(\boldsymbol{\omega}_{i, 0}, \boldsymbol{\omega}_{i, 1}, \ldots, \boldsymbol{\omega}_{i, 7}\right)$. The eight wrenches are generated by the eight vertices of the cuboid bounding the uncertainty:

$$
\boldsymbol{\omega}_{i, 0}=\left(\begin{array}{c}
\mathbf{f}_{i} \\
\left(\mathbf{p}_{i}-\boldsymbol{\eta}\right) \times \mathbf{f}_{i}
\end{array}\right), \boldsymbol{\omega}_{i, 7}=\left(\begin{array}{c}
\mathbf{f}_{i} \\
\left(\mathbf{p}_{i}+\boldsymbol{\eta}\right) \times \mathbf{f}_{i}
\end{array}\right) .
$$

The similiarity index $\gamma_{j, i}$ is then the volume of the intersection between the generated hypervolumes, calculated with the qhull library [18], normalized with the union of both volumes. 

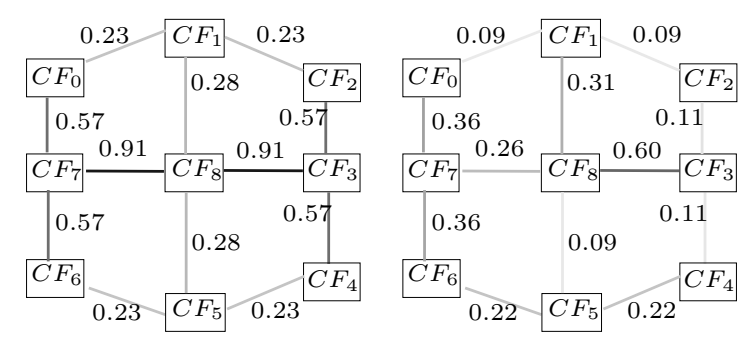

(a) Similarity index considering (b) Similarity index considering forces

forces and torques

Fig. 5. Contact formation graph with similarity index

Figure 5 shows the contact formation graph with the similarity indices for the example given in Section II. In Figure 5(a) only forces are considered, and in Figure 5(b) torques are taken into account as well. The darkness of the connection bars indicate the similarity of the wrench spaces. Note that the contact graph with similarity indices considering forces and torques $\gamma_{j, i}$ is not completely symmetric as the graph with indices $\gamma_{j, i}^{f}$. This is due to the geometry of the chosen object, as the distance between the contact points e.g. in $C F_{7}$ is a lot smaller than in $C F_{3}$ and the contact graph with similarity indices $\gamma_{j, i}^{f}$ does not take into account this information.

\section{Recognition of Contact Formations}

A particle filter can assign a likeliness to a contact formation given a measured force torque vector. The particle filter is a tool often used in localization for mobile robots dealing with uncertainties and sensor noise [19]. During assembly similar problems occur, making the particle filter an adequate tool for identifying contact formations.

In the following, a particle filter [20] with its measurement and system model is shortly described. Given a sequence of measured force torque vectors $\mathbf{z}_{0: k}$ from time step $t=0$ to $t=k$, the particle filter computes a probability to which contact formation each force torque vector belongs. This is done by comparing the wrench spaces (described in the previous section) with the force torque measurement. Note that the term "forces" always refers to the six dimensional force torque vector.

\section{A. Particle filter}

A particle filter constructs the state probability density function (pdf) $p\left(\mathcal{C F}_{k} \mid \mathbf{z}_{0: k}\right)$ at each time step $t(k)=t_{k}$, $k \in \mathbb{N}$. The pdf describes the belief in state $\mathcal{C F}_{k}$ at time $t_{k}$ given the measurements $\mathbf{z}_{0: k}$, with $\mathbf{z}_{k} \in \mathbb{R}^{6}$. The state $\mathcal{C} \mathcal{F}_{k}$ represents the contact formations, see Section II. The measurements $\mathbf{z}_{k}$ are the measured forces and torques, see Section VI. The pdf is approximated by $n_{p}$ particles, where the number of particles in each contact formation relates to the belief in this contact formation.

The calculation of the pdf is done recursively in two steps: the prediction and the update step. The procedure is explained for one contact formation $\mathcal{C F}_{k}(j)=C F_{k, j}$. During the prediction step, the probability $p\left(C F_{k, j} \mid \mathbf{z}_{0: k-1}\right)$ of a certain contact formation $C F_{k, j}$ given the previous measurements $\mathbf{z}_{0: k-1}$, is calculated. Given an initial pdf $p\left(C F_{0, j} \mid \mathbf{z}_{0}\right)=p\left(C F_{0, j}\right)$, the probability of the contact formation can be predicted based on the system model, see Section V-B, and the prior pdf $p\left(C F_{k-1, j} \mid \mathbf{z}_{0: k-1}\right)$ for $C F_{j}[6]$ :

$$
\begin{aligned}
& p\left(C F_{k, j} \mid \mathbf{z}_{0: k-1}\right)= \\
& \sum_{i=1}^{n_{C F}}\left[p\left(C F_{k, j} \mid C F_{k-1, i}\right) \cdot p\left(C F_{k-1, j} \mid \mathbf{z}_{0: k-1}\right)\right] .
\end{aligned}
$$

During the update step the predicted pdf (10) is corrected using the measurement model, see Section V-C, to calculate the pdf at time step $t_{k}$ :

$$
\begin{aligned}
& p\left(C F_{k, j} \mid \mathbf{z}_{0: k}\right)= \\
& p\left(\mathbf{z}_{k} \mid C F_{k, j}\right) \cdot p\left(C F_{k, j} \mid \mathbf{z}_{0: k-1}\right) .
\end{aligned}
$$

\section{B. The System Model}

The probability of a transition from contact formation $C F_{k-1, j}$ at time $t_{k-1}$ to $C F_{k, i}$ at $t_{k}$ is described by the system model which includes the information of the contact formation graph, see Section II. This means $p\left(C F_{k, j} \mid C F_{k-1, i}\right)=1$ if a transition exists between $C F_{i}$ and $C F_{j}$, and $p\left(C F_{k, j} \mid C F_{k-1, i}\right)=0$ if $C F_{i}$ and $C F_{j}$ are not neighbors in the graph.

\section{The Measurement Model}

The probability $p\left(\mathbf{z}_{k} \mid C F_{k, j}\right)$ of a measurement $\mathbf{z}_{k}$ given a contact formation $C F_{k, j}$ reflects the shortest distance $\mathbf{d}\langle\cdot\rangle$ between the measurement $\mathbf{z}_{k}$ and the possible wrenches of the contact formation. Hence, $p\left(\mathbf{z}_{k} \mid C F_{k, j}\right)$ is calculated as:

$$
p\left(\mathbf{z}_{k} \mid C F_{k, j}\right)= \begin{cases}\exp \left(-\mathbf{d}\left\langle\mathbf{z}_{k}, \boldsymbol{\omega}_{j}\right\rangle\right), & \text { if } \mathbf{z}_{k} \in \mathbf{f}_{j} \\ 0, & \text { else }\end{cases}
$$

where $\mathbf{f}_{j}$ represents the corresponding friction cone.

\section{Post-processing Step}

After the measured forces and torques are mapped to their corresponding contact formations by the particle filter, the similarity index is used to solve ambiguos assignments. As described in Section III, one measured force torque vector can map to several contact formations. Accordingly, the particle filter assigns beliefs to all the contact formations that the force torque vector can belong to. Whenever multiple contact formations for one force torque vector are possible, the similarity index, the transitions of the contact formation graph, and the history of already identified contact formations are considered.

To reduce the number of iteration steps, the time series of weights is roughly segmented. When the contact formation with the maximal weight changes, a cluster is formed. Each contact formation is then represented by the sum of its weights during that time period. The most likely combination of contact states is chosen by iterating through all clusters that have an assigned weight larger than zero. This is done first by removing all probabilities of contact formations 


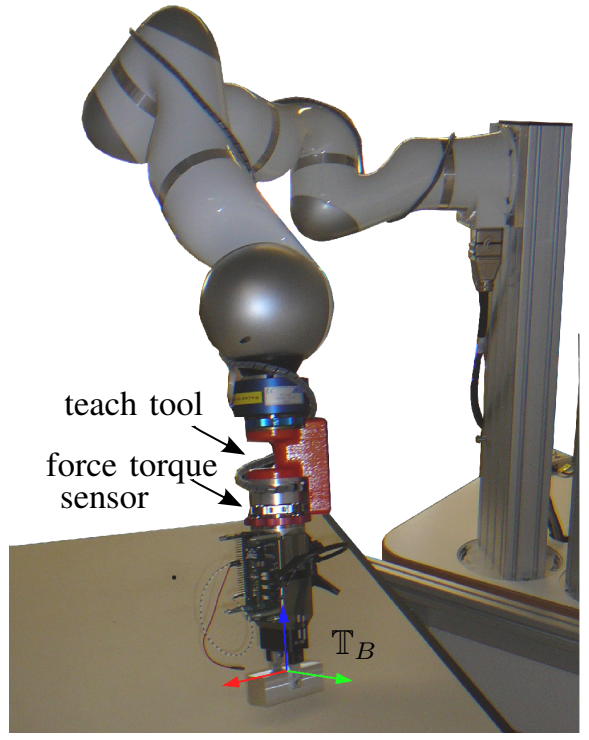

Fig. 6. Demonstration setup

that have no transitions to the previous identified contact. Secondly, the problem of maximizing the probability in contact states and minimizing the number of transitions is solved. Minimizing the number of transitions assumes that the human changes between contact formations only if necessary. For each contact formation, the similarity index to the other contact formations corresponds to the percentage their probability can be reduced for. Of all possible choices of contact formations, their modified weights, and their possible transitions, a weighted digraph is set up and solved for the shortest path.

The recognition of contact formations with the proposed particle filter and the post-processing step is evaluated in Section VI.

\section{EXPERIMENTS}

In previous sections, the theoretical background to identify contact formations based on measured forces and torques was introduced. This section describes first the demonstration setup and then reports on experiments executed to validate the presented approach.

A human demonstrates the assembly task by moving a DLR Light Weight Robot (LWR) [21] using a teach tool that is attached to the seventh joint of the robot, see Figure 6 . The robot arm is equipped with torque sensors, which enables backdriveable behavior and operation with gravity compensation. A force torque sensor attached to the teach tool measures the contact forces and torques

$$
S_{\mathbf{z}}=\left[\mathbf{f}_{\mathrm{msr}}, \boldsymbol{\tau}_{\mathrm{msr}}\right] \in \mathbb{R}^{6}
$$

between the manipulated object, which is grasped with a gripper, and the environment in the sensor frame $\mathbb{T}_{S}$. As the force torque sensor is located between the teach tool and the gripper, it measures only the contact forces independent of the forces that the human applies to the robot. The measures are also compensated for the mass and inertia of the gripper.
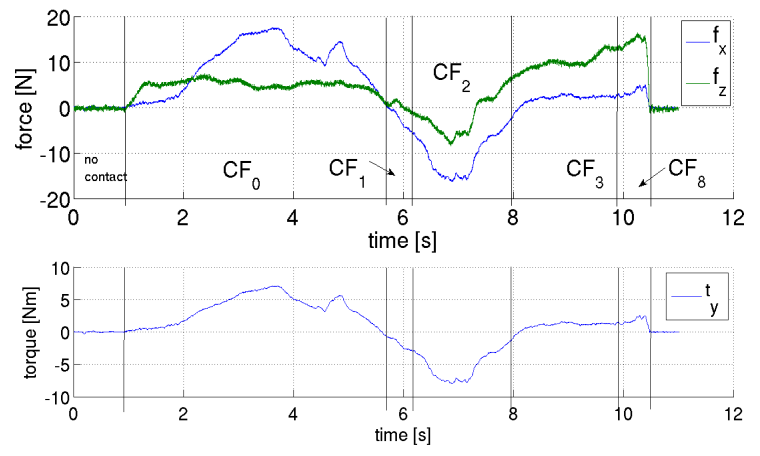

Fig. 7. Measured force torque vector for the 2-dimensional example

The measured forces are transformed into the object coordinate system $\mathbb{T}_{B}$, assuming a known grip transformation ${ }^{B} T_{G}$ from the gripper coordinate system $\mathbb{T}_{G}$ to the object $B$ :

$$
{ }^{B} \mathbf{z}=\left({ }^{B} T_{G} \cdot{ }^{G} T_{S}\right) \cdot{ }^{S} \mathbf{z},
$$

where ${ }^{G} T_{S}$ is the constant transformation from the sensor frame $\mathbb{T}_{S}$ to the gripper frame $\mathbb{T}_{G}$.

In summary, the demonstration provides the contact forces ${ }^{B} \mathbf{z}$ of object $B$ with the environment expressed in the object coordinate system $\mathbb{T}_{B}$. Note that no calibration of object poses is needed. The world coordinate system is unkown.

The similarity index is calculated approximating the resulting friction cone with $n_{f c}=60$ sides.

The 2D example of putting a rectangle in a corner is used to evaluate the particle filter. During the demonstration, the translation in $y$-direction is blocked. Forces are measured in $x$ and $z$ direction, the rotations are around the $y$-axis. The measured forces and torques are displayed in Figure 7. The state "no contact" between the objects can be seen before and after contact between the objects is established $(t<0.8 \mathrm{~s}$ and $t>10.5 s)$. The recognition of contact formation starts when $\left|\mathbf{f}_{\mathrm{msr}}\right|>1 N$. The sequence $C F_{0}, C F_{1}, C F_{2}, C F_{3}$, and $C F_{8}$ is demonstrated.

The probabilities assigned by the particle filter are shown in Figure 8. Note, that almost no contact formation has a high belief, which would correspond to a unique mapping of the force torque vector to a contact formation. Instead, contact formations are assigned similar probabilities, for example, during the first contact phase $C F_{0}, C F_{1}$ and $C F_{4}$ are candidates for a demonstrated contact formation. As the demonstrator then switches to $C F_{1}$, the particle filter computes probabilities for many contact formations. In the end, $C F_{8}$ is reached but only for about $0.1 s$.

This example makes clear that the particle filter alone is not sufficient to resolve ambiguous force torque information. In Figure 9 a simplified digraph for the post-processing step is depicted. One can see two transitions between three contact formations. Many other transitions and the weigths of the contact formations and transitions are omitted to improve the readability of the picture. The red marked formations have a high belief assigned. Between first and second row all possible transitions are shown, the red ones connect the 


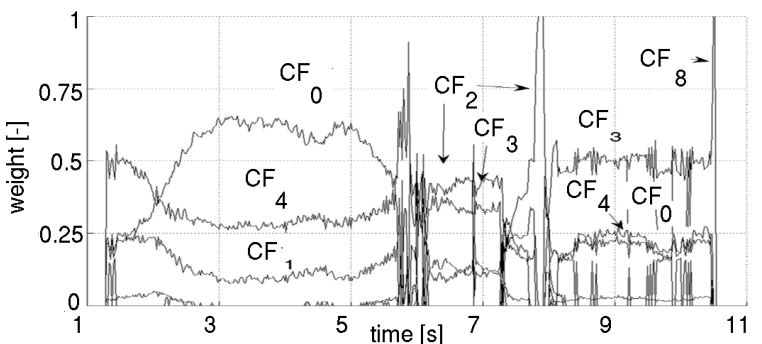

Fig. 8. Probabilities assigned by the particle filter

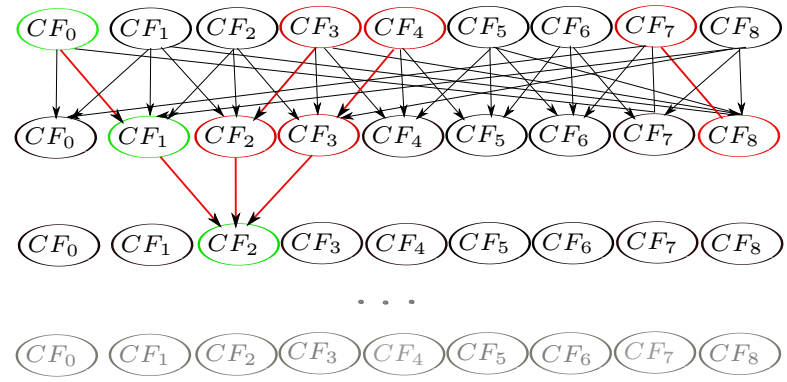

Fig. 9. Order of demonstrated contact formations

contact formations with high beliefs. The third row depicts time step $t=7.8 \mathrm{~s}$ where all beliefs are assigned to $C F_{2}$. This means that the prior contact formations can only be $C F_{1}, C F_{2}, C F_{3}$. Due to the distribution of probabilites of the contact formations, the demonstrated order of contact formations can be identified correctly (marked in green).

\section{CONCLUSIONS AND Future WORKS}

In this paper a new way of recognizing contact formations from human demonstration is proposed. The procedure can be divided in three steps: First, wrench spaces in the object coordinate frame are automatically computed based on a contact formation graph. Then, these spaces generate a similarity index that reflects the amount of overlap between the wrenches corresponding to different contact formations. Using a particle filter each measured force torque vector creates a likeliness of a contact formation. As one measurement can be assigned to several contact formations, this ambiguity is taken into account in a post-processing step. The approach is validated with experimental data.

In future works, the approach is transformed to an online recognition of contact states to be able to use it for automated assembly. The uncertainty of the grip transformation should be taken into account and soft contacts are going to be considered.

\section{REFERENCES}

[1] Y. Huang and C. Lee, "An automatic assembly planning system," in ICRA, pp. $1594-1599$ vol.3.
[2] H. Tönshoff, E. Menzel, and H. Park, "A knowledge-based system for automated assembly planning," CIRP Annals - Manufacturing Technology, vol. 41, no. 1, pp. 19 - 24, 1992.

[3] J. Xiao and X. Ji, "Automatic generation of high-level contact state space," The International Journal of Robotics Research, vol. 20, no. 7, pp. 584-606, 2001.

[4] U. Thomas, F. Wahl, J. Maass, and J. Hesselbach, "Towards a new concept of robot programming in high speed assembly applications," in Proc. IEEE/RSJ Int. Conf. on Intelligent Robots and Systems (IROS), aug. 2005, pp. $3827-3833$.

[5] W. Meeussen, J. Rutgeerts, K. Gadeyne, H. Bruyninckx, and J. D. Schutter, "Contact-state segmentation using particle filters for programming by human demonstration in compliant-motion tasks," IEEE Transactions on Robotics, vol. 23, no. 2, pp. 218 -231, apr. 2007.

[6] K. Gadeyne, T. Lefebvre, and H. Bruyninckx, "Bayesian hybrid modelstate estimation applied to simultaneous contact formation detection and geometrical parameter estimation," The International Journal of Robotics Research, vol. 24, pp. 615-630, 2005.

[7] M. Skubic and R. Volz, "Acquiring robust, force-based assembly skills from human demonstration," IEEE Transactions on Robotics and Automation, vol. 16, no. 6, pp. 772 -781, dec 2000.

[8] L. Everett, R. Ravuri, R. Volz, and M. Skubic, "Generalized recognition of single-ended contact formations," IEEE Transactions on Robotics and Automation, vol. 15, no. 5, pp. 829 -836, oct 1999.

[9] F. Abegg, A. Remde, and D. Henrich, "Force- and vision-based detection of contact state transitions," Universität Kaiserslautern, Tech. Rep., 2000.

[10] H. Mosemann, T. Bierwirth, F. Wahl, and S. Stöter, "Generating polyhedral convex cones from contact graphs for the identification of assembly process states," in Proc. of the IEEE Int. Conf. on Robotics and Automation (ICRA), 2000, pp. 744-749.

[11] S. Hirai and H. Asada, "Kinematics and statics of manipulation using the theory of polyhedral convex cones," The International Journal of Robotics Research, vol. 12, no. 5, pp. 434-447, 1993.

[12] R. Suárez, L. Basañez, and J. Rosell, "Assembly contact force domains in the presence of uncertainty," in Proc. of the Fourth IFAC Symposium on Robot Control, SYROCO'94. Pergamon Press/Elsevier ISBN 008-042227-6, september 1994, pp. 653-659.

[13] R. Suárez, L. Basanez, and J. Rosell, "Using configuration and force sensing in assembly task planning and execution," IEEE International Symposium on Assembly and Task Planning, 1995.

[14] B. J. McCarragher and H. Asada, "Qualitative template matching using dynamic process models for state transition recognition of robotic assembly," Journal of Dynamic Systems, Measurement, and Control, vol. 115, no. 2A, pp. 261-269, 1993.

[15] M. Sagardia, T. Hulin, C. Preusche, and G. Hirzinger, "Improvements of the voxmap-pointshell algorithm - fast generation of haptic datastructures," in 53rd Internationales Wissenschaftliches Kolloquium, September 2008.

[16] R. M. Murray, Z. Li, and S. Sastry, A Mathematical Introduction to Robotic Manipulation. Boca Raton, FL: CRC Press, 1994.

[17] I. N. Bronshtein, K. A. Semendyayev, G. Musiol, and H. Muehlig, Handbook of Mathematics, 5th ed. Springer, 2007.

[18] C. B. Barber, D. P. Dobkin, and H. Huhdanpaa, "The quickhull algorithm for convex hulls," ACM Transactions on Mathematical Software, vol. 22, no. 4, pp. 469-483, 1996.

[19] S. Thrun, "Particle filters in robotics," in Proceedings of the 17th Annual Conference on Uncertainty in AI (UAI), 2002.

[20] M. Arulampalam, S. Maskell, N. Gordon, and T. Clapp, "A tutorial on particle filters for on-line nonlinear/non-gaussian bayesian tracking," vol. 50, pp. 174-188, 2001.

[21] T. Hulin, K. Hertkorn, P. Kremer, S. Schätzle, J. Artigas, M. Sagardia, F. Zacharias, and C. Preusche, "The DLR bimanual haptic device with optimized workspace," in Proc. of the IEEE Int. Conf. on Robotics and Automation (ICRA), 2011, pp. 3441-3442. 